\title{
IDENTIFICAÇÃO GENÉTICA DE MODELOS POR PÓLOS E ZEROS BASEADA NO COMPROMISSO ENTRE OS ERROS DE POLARIZAÇÃO E VARIÂNCIA
}

\author{
L.V.R. Arruda* \\ arruda@cpgei.cefetpr.br
}

\author{
Sílvio Fávaro* \\ silvio.favaro@siemens.com.br
}

\author{
F. Neves-Jr.* \\ neves@cpgei.cefetpr.br
}

${ }^{*}$ CPGEI / CEFET-PR, Av. 7 de Setembro,3165, CEP 80230-901, Tel: 0xx41-3104689, Fax: 0xx41-3104686

Curitiba, PR, BRASIL

\begin{abstract}
This work proposes a genetic algorithm (GA) to solve process estimation problems when the real process presents high orders polynomials (complexity model) or non - linearities, non-minimum phase behavior, etc. The algorithm finds the best linear model in the pole and zero form to represent the real plant using its input and output signals. A new chromosome representation was introduced and a new "fitness" function based on the tradeoff bias $\mathrm{x}$ variance was developed. To validate this genetic estimator, simulations studies were done and the GA performance was compared with one obtained by use of the traditional least square estimation method.
\end{abstract}

KEYWORDS: Identification, Least Square estimation, genetic algorithm, reduced order model

\section{RESUMO}

Este trabalho propõem um algoritmo genético aplicado ao problema de identificação de plantas não-lineares, de fase não-mínima ou plantas lineares de ordem superior. $\mathrm{O}$ algoritmo proposto tem como objetivo encontrar um modelo linear na forma de pólos e zeros e de ordem re-

Artigo submetido em 20/12/00

1a. Revisão em 05/11/01; 2a. Revisão 04/02/02

Aceito sob recomendação do Ed. Assoc. Prof. Jacques Szczupak duzida, que melhor represente a planta real, a partir dos sinais de entrada e saída. Uma proposta inovadora para a representação dos indivíduos e função de "fitness" foi desenvolvida neste trabalho. Esta função de fitness representa o compromisso entre os erros de polarização e variância do modelo estimado. Já a representação proposta divide o espaço de busca em dois subespaços: um destinado à busca de pólos e zeros de natureza real e outro com a finalidade de se encontrar os pólos e zeros complexos. Resultados de simulação são utilizados para ilustrar o desempenho do estimador genético desenvolvido.

PALAVRAS-CHAVE: Identificação, Mínimos Quadrados, Algoritmo genético, modelos de ordem reduzida.

\section{INTRODUÇÃO}

A complexidade dos sistemas industriais atualmente torna as tarefas dos engenheiros e projetistas responsáveis pela identificação, controle e supervisão cada vez mais difícil, uma vez que as ferramentas matemáticas tradicionais são deficientes em modelar algumas características típicas destes sistemas tais que não-linearidades, atrasos variantes no tempo, perturbações não brancas, etc. (Tan et alli., 1995).

No caso específico de modelagem de sistemas para fins 
de controle, esta complexidade causa problemas na escolha da melhor estrutura para o modelo que se deseja representar. As técnicas convencionais de estimação, (por exemplo: mínimos quadrados e máxima verossimilhança) costumam falhar quando, a fim de diminuir a complexidade do sistema a ser controlado, opta-se por um modelo de ordem reduzida em relação ao sistema real. Isto porque a maior parte destas técnicas é baseada no método do gradiente, e facilmente convergem para mínimos locais, levando a modelos errôneos que colocarão em risco toda a estrutura de controle que for projetada a partir do modelo identificado (Kristisson e Dumont, 1992).

Motivado por esta discussão, o objetivo deste trabalho é propor uma metodologia baseada nos algoritmos genéticos (AG) capaz de fornecer um modelo simplificado (linear e de ordem reduzida) para processos complexos, facilitando assim a síntese de controladores, mas sendo capaz de reproduzir, o melhor possível, o comportamento temporal destes processos e suas características freqüenciais. A utilização de AG que é uma classe de algoritmos de busca estocástica, baseada nos conceitos de seleção natural e genética (Michalewicz, 1996), é uma técnica indicada para a aplicação em problemas de otimização, quando o espaço de busca é multimodal e extenso, como é o caso dos problemas acima citados (Tan et alli.1995).

A idéia básica da identificação de sistemas é permitir a construção de modelos matemáticos de um sistema dinâmico baseado em medidas, efetuando ajustes de parâmetros para um dado modelo até que a saída coincida tão bem quanto possível com as saídas medidas (Ljung, 1999). Desde que os parâmetros do sistema real minimizam uma função objetivo para um conjunto de dados de entrada e saída, a aplicação de um algoritmo genético para identificação é possível. O objetivo que o identificador genético procura otimizar (função de fitness do AG) é um conjunto de parâmetros de um dado modelo que mais se aproxime do sistema real. Assim sendo, o algoritmo genético pode tratar cada conjunto de parâmetros de um modelo como sendo um indivíduo. Estes indivíduos compõem uma população que ao longo das gerações converge para um indivíduo ótimo que é o melhor modelo capaz de representar o sistema real. Uma revisão bibliográfica sobre a aplicação de AG na identificação de modelos de processos pode ser encontrada em (Favaro, 1999) e (Coelho e Coelho, 1999).

Quando o objetivo do modelo identificado é a síntese de controladores, a função de transferência do sistema pode ser representada por seus pólos e zeros. No entanto a identificação direta de pólos e zeros de funções de transferência constitui um problema de estimação não-linear que requer a utilização de métodos mais elaborados tais que os métodos de Levenberg-Marquadt e Gauss-Newton, entre outros. Estes métodos são difíceis de utilizar já que requerem o cálculo analítico das derivadas de primeira e segunda ordem da função custo, o que pode ser impraticável para sistemas de ordem maior que dois, ou para sistemas com função custo não diferenciável no espaço de parâmetros. Os estimadores obtidos apresentam convergência lenta e a implementação do método como um todo requer um grande esforço computacional (Lindskorg, 1997). O método proposto neste trabalho deve superar estas dificuldades, gerando um algoritmo de estimação em que o usuário não precise se preocupar com cálculos intermediários que dependem da ordem do modelo, nem com o mau condicionamento da matriz hessiana da função custo, nem com a convergência lenta dos parâmetros. Além disso, o estimador genético proposto deve estimar, a partir de um conjunto de dados de entrada e saída do sistema, um modelo de pólos e zeros de menor ordem admissível, segundo um critério de qualidade que leva em conta o compromisso entre os erros de polarização e de variância, calculados entre o sistema real e o modelo estimado (Ninness e Goodwin, 1995).

Este artigo é organizado da seguinte forma. A seção 2 descreve o algoritmo genético padrão proposto por (Holland, 1975) que será utilizado como base neste trabalho. Os aspectos relacionados à modelagem e identificação de sistemas, bem como os critérios utilizados para seleção do modelo estimado são discutidos na seção 3 . A representação utilizada pelo AG, assim como o espaço de busca, a função de fitness considerada, a estratégia de seleção e os operadores genéticos utilizados são apresentados na seção 4 . Na seção 5 , o desempenho do estimador genético proposto é estudado a partir de resultados de simulação. As conclusões e perspectivas são apresentadas na seção 6 .

\section{ALGORITMOS GENÉTICOS}

De um modo geral, os Algoritmos Genéticos tentam imitar o princípio de Darwin da seleção natural: um indivíduo adaptado de uma população tende a reproduzir e transmitir seus genes para a próxima geração, tornando as gerações seguintes cada vez melhores. Algoritmos genéticos têm provado que são uma ferramenta de otimização eficiente e flexível que pode encontrar a solução ótima ou sub-ótima para problemas lineares ou não-lineares, pela exploração simultânea de várias regiões do espaço. Esta exploração das áreas promissoras é feita exponencialmente através de operadores de seleção, mutação e cruzamento. Diferentemente de outras 
técnicas de otimização, algoritmos genéticos não exigem condições favoráveis das funções de otimização para que possam ser aplicados (Michalewicz, 1996).

O Algoritmo genético utilizado neste artigo foi proposto em (Holland, 1975) onde cada solução ou indivíduo da população é descrito por um vetor de variáveis em ponto flutuante (representação dos cromossomos). O primeiro passo do AG é inicializar a população aleatoriamente ou por "seeding". Uma vez que a população inicial foi gerada, cada indivíduo, $i$, é avaliado através da função objetivo para se determinar o seu "fitness" ou valor, $F_{i}$. Um subconjunto da população é selecionado para produzir a próxima geração. Um indivíduo da população pode ser selecionado mais de uma vez. Uma seleção probabilística é executada, sendo que os indivíduos com maior "fitness" têm mais chance de serem selecionados. Os indivíduos selecionados se reproduzem através dos operadores genéticos para produzir uma nova população. O AG evolui de geração em geração até que algum critério de parada seja encontrado.

$\mathrm{Na}$ construção de um algoritmo genético, seis fatores fundamentais afetam seu desempenho e precisam ser considerados: a representação dos cromossomos, a inicialização da população, a função de "fitness", a estratégia de seleção, os operadores genéticos e o critério de parada. Estes fatores serão apresentados na seção 4 a seguir.

\section{MODELAGEM E IDENTIFICAÇÃO DE POLOS E ZEROS}

Considere um sistema descrito pelo modelo ARX, dado na equação (1),

$$
A\left(q^{-1}\right) y(t)=B\left(q^{-1}\right) u(t-d)+e(t)
$$

onde, $A\left(q^{-1}\right), B\left(q^{-1}\right)$ são polinômios mônicos representados através do operador deslocamento $q^{-1}, d$ é o atraso de transporte, e os sinais $y(t), u(t)$ e $e(t)$ representam respectivamente a saída, a entrada e o ruído. O ruído é uma seqüência aleatória normalmente distribuída com média zero e variância $\left(\sigma_{e}^{2}\right)$. O objetivo da identificação é obter os polinômios $A\left(q^{-1}\right)$ e $B\left(q^{-1}\right)$ a partir dos dados de entrada $u(t)$ e saída $y(t)$ (Ljung, 1999).

Uma seqüência $\eta(t)$ pode ser definida para se observar quão bem o modelo representa o sistema real, isto é, para medir o "erro mismatch". A definição matemática deste sinal pode ser vista nas equações (2) e (3).

$$
\begin{gathered}
\eta(t)=y(t)-\hat{y}(t) \\
\hat{A}\left(q^{-1}\right) \hat{y}(t)=\hat{B}\left(q^{-1}\right) \cdot u(t-d)
\end{gathered}
$$

onde $\hat{y}(t)$ é a saída de um sistema determinístico obtida a partir do modelo quando aplicada a entrada $u(t)$, e $\hat{A}\left(q^{-1}\right)$ e $\hat{B}\left(q^{-1}\right)$ são as estimativas de $A\left(q^{-1}\right)$ e $B\left(q^{-1}\right)$ encontradas para minimizar o critério escolhido. A equação (2) representa o erro previsto um passo a frente entre a saída real e a saída estimada.

Comparando as equações (1) e (3) tem-se que o "erro mismatch" é formado por duas parcelas: uma, denominada erro de polarização, é devido ao fato que o sistema real não pode ser representado integralmente pela estrutura do modelo escolhido (por exemplo: as ordens dos polinômios estimados são menores que a ordem real dos polinômios $A\left(q^{-1}\right)$ e $B\left(q^{-1}\right)$ ); a outra parcela é devida à presença do ruído e(t) sobre os dados observados. Esta é conhecida como erro de variância. Em (Ljung, 1985), é mostrado que o erro de polarização diminui com o aumento da ordem do modelo, desde que a generalização da estrutura do modelo é aumentada. No entanto, o erro de variância aumenta com a ordem do modelo, apesar dele diminuir com o número de dados observados. O resultado disso é que, a partir de um conjunto de dados de tamanho já conhecido, há uma ordem de modelo ótima que equilibra a diminuição do erro de polarização com o aumento do erro de variância e permite obter o menor erro entre o modelo estimado e o sistema. A figura 1 ilustra o comportamento destes dois erros em relação ao erro total de estimação (Ninness e Goodwin, 1995).

Este equilíbrio é conhecido na literatura como o "tradeoff" entre variância e polarização (Ljung, 1985). Observa-se na figura 1 que o erro de polarização diminui com o aumento da complexidade da estrutura. Por outro lado, o aumento desta complexidade produz um aumento do erro de variância. Para encontrar esta ordem ótima, quando se utilizam algoritmos clássicos do tipo mínimos quadrados é necessário executar várias vezes o estimador com diferentes estruturas (ordens do polinômio A e B), obtendo diferentes modelos. Em seguida deve ser construído o gráfico do erro previsto para estes modelos, a fim de se determinar visualmente o ponto de inflexão da curva, correspondendo à ordem ótima. Já o estimador genético proposto neste artigo, busca diretamente o ponto mínimo mostrado na figura 1, identificando ao mesmo tempo a estrutura (ordem ótima) e os parâmetros (pólos e zeros) do modelo.

A função de "fitness" utilizada e que será apresentada na seção 4, é capaz de encontrar este equilíbrio, minimizando o erro total entre o modelo e o sistema real.

Por outro lado, considerando o modelo ARX dado na equação (1), as estimativas dos polinômios $A\left(q^{-1}\right)$ e 


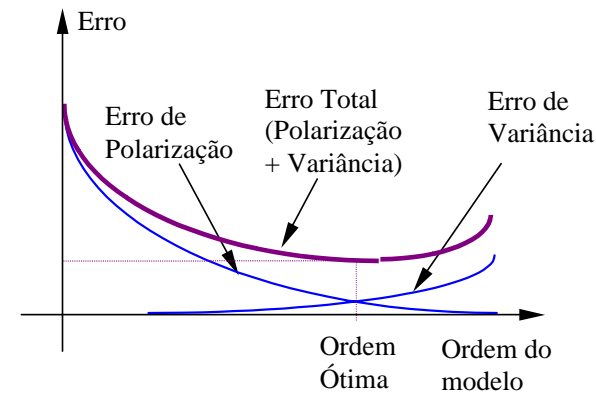

Figura 1: Compromisso entre os erros de polarização e variância

$B\left(q^{-1}\right)$ podem ser representadas em uma forma fatorada conforme as equações (4) e (5):

$$
\begin{aligned}
& A\left(q^{-1}\right)=\left(1-p_{1} \cdot q^{-1}\right) \cdot\left(1-p_{2} \cdot q^{-1}\right) \ldots\left(1-p_{n} \cdot q^{-1}\right) \\
& B\left(q^{-1}\right)=\left(1-z_{1} \cdot q^{-1}\right) \cdot\left(1-z_{2} \cdot q^{-1}\right) \ldots\left(1-z_{m} \cdot q^{-1}\right)
\end{aligned}
$$

Onde:

$p_{i}=$ pólo de $A(q), i=1,2, \ldots n$

$z_{j}=$ zero de $B(q), j=1,2, \ldots m$

$n=$ n máximo de pólos de $A\left(q^{-1}\right)$

$m=$ n máximo de zeros de $B\left(q^{-1}\right)$

A partir da equação (3) pode-se definir a função de transferência na forma de pólos e zeros como:

$$
G\left(q^{-1}\right)=\frac{\left(1-z_{1} \cdot q^{-1}\right) \cdot\left(1-z_{2} \cdot q^{-1}\right) \ldots\left(1-z_{m} \cdot q^{-1}\right)}{\left(1-p_{1} \cdot q^{-1}\right) \cdot\left(1-p_{2} \cdot q^{-1}\right) \ldots\left(1-p_{n} \cdot q^{-1}\right)}
$$

Um fato importante a se ressaltar é que os pólos e zeros anteriormente descritos podem ser números reais ou complexos. No caso de pólos ou zeros complexos, sabese que a existência de um pólo ou zero complexo implica necessariamente na existência de seu conjugado.

A obtenção de maneira direta dos pólos e zeros do modelo é uma vantagem que o identificador genético proposto apresenta, uma vez que outros métodos paramétricos tais que o Algoritmo dos Mínimos Quadrados calculam diretamente os coeficientes dos polinômios $A\left(q^{-1}\right)$ e $B\left(q^{-1}\right)$, sendo necessários em seguida fatorá-los para obter os pólos e zeros. Outra solução, como discutido anteriormente, seria empregar métodos de estimação nãolineares que são mais complexos e de difícil convergência.

\section{ESTIMADOR GENÉTICO DESENVOL- VIDO}

Os principais aspectos inovadores do algoritmo genético de estimação proposto neste trabalho são o espaço de busca, a representação utilizada e a função de fitness desenvolvida. Estes aspectos estão diretamente relacionados à qualidade do modelo obtido: o espaço de busca fornece a precisão do modelo estimado, a representação proposta confere ao estimador versatilidade em termos de estrutura de modelos, e por fim a função de fitness que mapeia o compromisso entre os erros de polarização e variância, deve garantir que o modelo estimado é de ordem ótima, segundo os critérios discutidos no item 3 anterior.

\subsection{Representação dos indivíduos}

Neste trabalho serão considerados somente sistemas discretos e estáveis, portanto pode-se inferir que os pólos estarão contidos dentro de um círculo unitário. Considere primeiramente que o espaço de busca será o quadrado tracejado ABCD como mostrado n a figura 2.

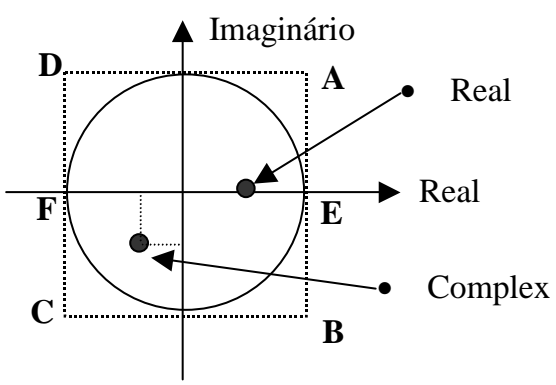

Figura 2: Espaço de Busca

Este espaço será dividido em dois subespaços: o primeiro é formado pelo retângulo EBCF e é destinado à busca de pólos e zeros complexos; o segundo subespaço é formado pelo retângulo AEFD e é destinado à busca de pólos e zeros reais. Neste caso, se desprezará a parte imaginária na composição dos pólos e zeros reais da função de transferência.

Esta representação proposta apresenta a desvantagem de utilizar um espaço de busca maior quando comparado com a representação cartesiana convencional, porém permite um equilíbrio na busca entre os indivíduos com características reais e complexas, fato que não ocorre com a representação convencional. A partir disto, foi proposto como modelo a ser usado na identificação via algoritmo genético a equação (7), a seguir (Favaro, 1999):

$G\left(q^{-1}\right)=K \cdot \frac{\left[1-m_{1} \cdot\left(a_{1} \pm j \cdot b_{1}\right) \cdot q^{-1}\right] \cdot\left[1-m_{2} \cdot\left(a_{2} \pm j \cdot b_{2}\right) \cdot q^{-1}\right]}{\left[1-m_{3} \cdot\left(c_{1} \pm j \cdot d_{1}\right) q^{-1}\right] \cdot\left[1-m_{4} \cdot\left(c_{2} \pm j \cdot d_{2}\right) \cdot q^{-1}\right]}$

onde

$K=$ ganho em malha aberta do sistema 
$a_{i}=$ parte real do i-ésimo zero

$b_{i}=$ parte imaginária do i-ésimo zero

$c_{i}=$ parte real do i-ésimo pólo

$d_{i}=$ parte imaginária do i-ésimo pólo

$m_{l}=$ multiplicador do l-ésimo pólo / zero

$z_{i}=i$-ésimo zero

$p_{i}=i$-ésimo pólo

$i=$ índice para os pólos / zeros

$l=$ índice para os multiplicadores dos pólos / zeros

O modelo aqui proposto será representado como um indivíduo da população (família de modelos) da seguinte forma: Indivíduo $\rightarrow\left(K, a_{1}, b_{1}, a_{2}, b_{2}, c_{1}, d_{1}, c_{2}, d_{2}, m_{1}, m_{2}\right.$,
$\left.m_{3}, m_{4}\right)$

Neste modelo duas famílias de pólos e duas de zeros são possíveis. Cada família pode ser constituída por um pólo ou zero real ou por um par complexo. Os multiplicadores " $m_{i}$ " foram introduzidos com intuito de modificar a estrutura do modelo para que esta se adapte mais à estrutura do processo real.

Para este modelo, o espaço de busca deve ser considerado como sendo o interior do quadrado $\mathrm{ABCD}$ na figura 2 , definido no plano " $z$ ", cujos limites no eixo real são -1 e 1 e no eixo imaginário são -1 e 1 . O tratamento que se dará aos pólos que se encontrem fora do círculo unitário (comportamento instável), bem como o tratamento de modelos fisicamente não realizáveis será a atribuição de um coeficiente de fitness punitivo, assim estes indivíduos serão naturalmente eliminados ao longo das gerações. O modelo apresentado anteriormente apresenta as seguintes particularidades:

- A ordem do modelo varia com a natureza real ou complexa dos pólos / zeros.

- Os multiplicadores $\left(m_{i}\right)$ permitem variações da estrutura do modelo

- A ordem mínima possível é 1 ( 1 pólo real)

- A ordem máxima possível é 4 (2 pares de pólos complexos)

\subsection{Espaço de Busca}

A partir do modelo anteriormente apresentado, pode-se avaliar o tamanho do espaço de busca. Escolhendo-se, por exemplo, uma resolução, $\varepsilon$, de 0,0001 e considerando os limites do espaço de busca de cada parâmetros como segue:

$$
\begin{aligned}
& a_{i} \subset\left[\begin{array}{ll}
-1 & 1
\end{array}\right] \\
& b_{i} \subset\left[\begin{array}{ll}
-1 & 1
\end{array}\right] \\
& c_{i} \subset\left[\begin{array}{ll}
-1 & 1
\end{array}\right] \\
& d_{i} \subset\left[\begin{array}{ll}
-1 & 1
\end{array}\right] \\
& K \subset\left[\begin{array}{ll}
0.0001 & 20
\end{array}\right] \\
& m_{l} \subset\{0,1\}
\end{aligned}
$$

É possível determinar a dimensão do espaço de busca com sendo:

$$
\begin{aligned}
\text { Dimensão } & \cong\left(2 \times 10^{4}\right)^{4} \times\left(2 \times 10^{4}\right)^{4} \times\left(2 \times 10^{5}\right) \times 2^{4} \\
& \cong 2^{13} \times 10^{37}
\end{aligned}
$$

Ou seja, isto nos leva a um espaço de busca de aproximadamente $8,19 \times 10^{41}$ alternativas.

\subsection{Função de Fitness}

A função de "fitness" aqui proposta pode ser vista na equação (8):

$$
F=\frac{1}{2}\left(e^{-a \cdot \sqrt{\sum_{i=1}^{w}\left(\eta_{i}\right)^{2}}}+e^{-b \cdot\left|1-C_{x y}\right|}\right)
$$

Esta função apresenta duas parcelas. A primeira parcela do numerador é a função de "fitness" proposta em (Tan et alli., 1995) onde "a"é um fator de escalonamento que permite ajustar o decaimento da função exponencial, $w$ é o número de amostras, $\eta$ é o erro previsto dado em (2). $\mathrm{O}$ ajuste do fator de escalonamento é muito importante, pois, se este for muito alto, o decaimento será muito acentuado, permitindo que os indivíduos da população inicial tenham coeficiente de "fitness" próximo de zero. Isto compromete a geração dos novos indivíduos e pode levar a problemas de convergência do AG. Por outro lado, se o fator de escalonamento for muito pequeno, o decaimento será pouco intenso e a diferenciação entre os indivíduos será muito difícil, pois uma grande variação no erro será representada por uma pequena variação no coeficiente de fitness. Neste trabalho, o valor de $a$ foi definido empiricamente como $5 \times 10^{-4}$, o que permitiu obter-se um comportamento ideal para o decaimento da parcela exponencial da função de fitness.

Já a segunda parcela na equação (8) foi desenvolvida neste trabalho, para modelar o comportamento aleatório do erro previsto. Nesta função, $b=10$ é um segundo fator empírico de escalonamento, e $C_{x y}$ é o coeficiente de correlação entre a saída real do sistema e a saída obtida pelo modelo. A idéia de se utilizar este coeficiente de correlação junto a função de "fitness" se deve ao seguinte fato: se a saída real e a simulada forem idênticas, 
o coeficiente de correlação será unitário, tornando a segunda parcela unitária (Favaro, 1999). Os valores de " $a$ " e " $b$ " foram mantidos para todos os casos estudados neste trabalho.

A partir da equação (8), vê-se que a primeira parcela representa o erro de polarização associado ao estimador, e a segunda parcela representa o erro de variância. Desta forma, o algoritmo genético ao procurar por um indivíduo que minimize esta função de fitness, está na verdade buscando o ponto de equilíbrio mostrado na figura 1. Observou-se também através de simulações que a primeira parcela tem a função de permitir uma boa identificação do ganho de regime do sistema, além de auxiliar na busca dos pólos e dos zeros do sistema; já a segunda parcela tem a função de auxiliar na identificação exclusivamente dos pólos e zeros, uma vez que esta é mais sensível a eles.

\subsection{Aspectos Gerais do AG}

A plataforma de software utilizada para o desenvolvimento do algoritmo foi o MATLAB ${ }^{1}$ com auxílio de uma biblioteca específica para algoritmos genéticos (Joines, 1998). Alguns dos parâmetros principais que foram utilizados neste algoritmo podem ser visto na tabela (1).

Tabela 1: Parâmetros gerais do AG.

\begin{tabular}{|c|c|}
\hline Parâmetros Gerais & Valores \\
\hline Tamanho da População & 150 \\
\hline N máximo de Gerações $^{\circ}$ & 400 \\
\hline Precisão Numérica & 0,000001 \\
\hline Discretização do Espaço & 0,0001 \\
\hline
\end{tabular}

A população inicial do algoritmo genético utilizada neste trabalho foi inicializada de maneira aleatória ao longo do espaço de busca. E o critério de parada adotado foi: o melhor indivíduo da população atingiu o "fitness" máximo ou o número máximo de gerações especificado foi atingido. $\mathrm{O}$ que ocorrer primeiro determina o término do AG.

\subsection{Estratégia de seleção}

O método de seleção utilizado neste trabalho foi o "Ranking" Geométrico Normalizado que segundo (Joines 1999) evita a proliferação de super indivíduos e diminui a pressão seletiva que causam problemas na convergência do algoritmo. Nos métodos do tipo "ranking", os indivíduos da população são selecionados com base em

\footnotetext{
${ }^{1}$ MatLab é marca registrada de MathWorks, Inc.
}

seus "ranks" na população (i.e., quão bom são eles comparados com o restante da população). Neste método, o AG não se preocupa com a diferença absoluta entre os indivíduos (i.e., pode-se falar que o indivíduo A é melhor que o indivíduo $\mathrm{B}$, mas não quanto melhor o indivíduo é). Os indivíduos da população são classificados do melhor para o pior de acordo com seu valor de "fitness". A cada indivíduo é associada uma probabilidade de seleção proporcional a sua posição ("rank"), baseada em alguma distribuição linear ou não linear.

No método do "Ranking" Geométrico Normalizado, a distribuição utilizada força a soma das probabilidades de seleção dos indivíduos a ser igual à unidade:

$$
\begin{gathered}
P[\text { seleção do indiv. } r]=q^{\prime}(1-q)^{r-1} \\
q^{\prime}=\frac{q}{1-(1-q)^{N}}
\end{gathered}
$$

onde

$q=$ probabilidade de selecionar o melhor indivíduo.

$r=$ "ranking" de cada indivíduo, onde o melhor tem valor 1 .

$N=$ tamanho da população.

Métodos baseados no "ranking" freqüentemente apresentam algumas vantagens sobre o método da roleta (Michalewicz, 1996). A população precisa somente ser ordenada do melhor para o pior indivíduo. A pressão seletiva pode ser controlada a partir do valor da probabilidade $q$. Porém não existem regras para se determinar o valor mais apropriado para esta probabilidade. Neste trabalho, a probabilidade de seleção do melhor indivíduo " $q$ " escolhida foi de 0,005 .

\subsection{Operadores genéticos}

Os operadores genéticos são os mecanismos de busca básicos do AG que são usados para criar novas soluções baseadas nas soluções existentes na população atual. Existem dois tipos básicos de operadores: cruzamento e mutação. O cruzamento seleciona dois indivíduos e produz dois novos indivíduos enquanto a mutação altera um indivíduo para produzir uma nova solução. A aplicação destes dois tipos básicos depende da representação dos cromossomos utilizados.

Os operadores genéticos utilizados foram: mutação uniforme, mutação não uniforme, multi mutação não uniforme, mutação de contorno, cruzamento simples, cruzamento aritmético e cruzamento heurístico, implementados de acordo com a biblioteca acima citada (Joines, 1998).

A frequência de aplicação dos operadores genéticos é um parâmetro muito importante no funcionamento do 
AG. Ele define quantas vezes será aplicado cada operador genético na população corrente, a fim de se gerar a população seguinte. Se a freqüência de aplicação de operadores de cruzamento for muito elevada, isto implicará em uma convergência prematura possivelmente para um máximo/mínimo local. Por outro lado, se a freqüência de aplicação de mutação for muito alta, a busca será quase que aleatória, implicando em uma convergência muito lenta. Assim sendo, deve-se ter um equilíbrio na aplicação destes operadores a fim de se evitar os problemas acima descritos.

As freqüências utilizadas neste trabalho foram definidas empiricamente. A fim de se observar a influência de cada operador sobre o problema, foram realizadas várias simulações. Nestas simulações foram utilizados dois operadores de cruzamento e dois de mutação. As várias combinações possíveis foram testadas e as melhores freqüências observadas são aquelas dadas na tabela 2 a seguir (Fávaro, 1999).

Para os operadores mutação não uniforme, multi mutação não uniforme e cruzamento heurístico os parâmetros que foram utilizados são dados na tabela 3 , onde $B$ é o parâmetro de forma, $G_{\max }$ o número máximo de gerações e $t$ o número de falhas permitido, conforme descrito em (Joines, 1998) e (Michalewicz, 1996).

Tabela 2: Freqüência de aplicação dos operadores.

\begin{tabular}{|c|c|}
\hline Operadores de Cruzamento & Freqüência \\
\hline Simples & $10 \%$ \\
\hline Aritmético & $10 \%$ \\
\hline Heurístico & $33,3 \%$ \\
\hline Total de Cruzamento & $53,3 \%$ \\
\hline Operadores de Mutação & Freqüência \\
\hline Contorno & $3,3 \%$ \\
\hline Uniforme & $3,3 \%$ \\
\hline Não Uniforme & $6,6 \%$ \\
\hline Multi Não Uniforme & $6,6 \%$ \\
\hline Total de Mutação & $19,8 \%$ \\
\hline
\end{tabular}

Tabela 3: Outros operadores utilizados.

\begin{tabular}{|c|c|}
\hline Operador & Valor \\
\hline Mutação não uniforme & $\mathrm{B}=3$, Gmax $=400$ \\
\hline Multi mutação não uniforme & $\mathrm{B}=3, \mathrm{Gmax}=400$ \\
\hline Cruzamento heurístico & $\mathrm{t}=3$ \\
\hline
\end{tabular}

\section{RESULTADOS DE SIMULAÇÃO}

A fim de avaliar o desempenho do estimador genético, foram realizadas comparações da resposta real do sistema, com a resposta fornecida pelo sistema identifi- cado através do AG e com a resposta obtida pelo tradicional método dos Mínimos Quadrados. Apesar do método proposto não ser exatamente uma técnica alternativa ao método dos Mínimos Quadrados, e sim aos métodos não-lineares de estimação, optou-se por uma comparação com os Mínimos Quadrados, por este ser um método de fácil implementação e largamente difundido na comunidade de controle adaptativo. Neste caso, o estimador MQ é aplicado a um modelo de regressão linear em que são estimados os coeficientes dos polinômios $A\left(q^{-1}\right), B\left(q^{-1}\right)$, e em seguida são calculados os pólos e zeros da função de transferência obtida. Também foram realizadas comparações gráficas da resposta no domínio da freqüência através dos diagramas de Bode de módulo do sistema real e dos modelos estimados (genético e mínimos quadrados).

\section{$1^{\circ}$ Caso: Planta Ordem Superior ao Modelo}

A planta escolhida é de $6^{a}$ ordem e apresenta um atraso de 5 , conforme indicado em (11).

O sinal de entrada utilizado é uma PRBS de amplitude unitária. As respostas temporal e freqüencial do sistema real e dos modelos obtidos com os estimadores MQ e AG são dadas nas figuras 3 e 4, respectivamente. Os valores de média e variância do erro mismatch são dados na tabela 4. O modelo identificado pelo AG é descrito em (12). Já o identificador dos mínimos quadrados obteve o modelo apresentado em (13).

\section{$2^{\circ}$ Caso: Planta Não Linear Fase Não Mínima}

A planta escolhida apresenta uma não linearidade do tipo saturação com limites em $\pm 0,8$. O sinal de entrada utilizado é uma PRBS de amplitude unitária. O erro mismatch produzido pelos estimadores MQ e AG são dados na figura 5. Os valores de média e variância representativos do compromisso dado pelo fitness são apresentados na tabela 4 . As respostas temporais produzidas pelo sistema real, mínimos quadrados (MQ) e estimador genético (AG) são dadas em figura 6 .

É sabido que um modelo linear será incapaz de representar o sistema real que é não-linear. Neste caso, espera-se que os modelos lineares identificados sejam os que melhor aproximem o comportamento do sistema não linear. $\mathrm{O}$ identificador genético obteve o modelo descrito por (14). Já o identificador dos mínimos quadrados obteve o modelo apresentado em (15).

\subsection{Análise dos Resultados}

No $1^{\circ}$ caso, como esperado, o modelo gerado pelo estimador MQ não foi capaz de representar todas as freqüên- 


$$
\begin{aligned}
G\left(z^{-1}\right) & =\frac{0,01 \cdot z^{-5}+0,0074 \cdot z^{-6}+0,000924 \cdot z^{-7}-0,00001762 \cdot z^{-8}}{1-2,14 z^{-1}+1,3049 z^{-2}+0,0963 z^{-3}-0,3467 z^{-4}+0,1097 z^{-5}-0,0105 z^{-6}} \\
G_{A G} & =\frac{0,055}{\left[1-(0,7064 \pm 0,3111 \cdot j) \cdot z^{-1}\right] \cdot\left[1-(0,8678 \pm 0,0641 \cdot j) \cdot z^{-1}\right]} \\
G_{M Q} & =\frac{\left(1+7,9210 \cdot z^{-1}\right) \cdot\left(1+1,0607 \cdot z^{-1}\right) \cdot\left(1-1,2647 \cdot z^{-1}\right)}{\left[1-(0,6150 \pm 0,4297 \cdot j) \cdot z^{-1}\right] \cdot\left[1-(0,8785 \pm 0,1224 \cdot j) \cdot z^{-1}\right]} \\
G_{A G} & =\frac{0,214}{\left[1-(0,5352 \pm 0,5186 \cdot j) \cdot z^{-1}\right] \cdot\left[1-(0,6485 \pm 0,1494 \cdot j) \cdot z^{-1}\right]} \\
G_{M Q} & =\frac{\left(1+1,8072 \cdot z^{-1}\right) \cdot\left[1+(0,0382 \pm 1,1641 \cdot j) \cdot z^{-1}\right]}{\left[1-(0,2890 \pm 0,3658 \cdot j) \cdot z^{-1}\right] \cdot\left[1-(0,5487 \pm 0,0842 \cdot j) \cdot z^{-1}\right]}
\end{aligned}
$$

Tabela 4: Compromisso entre polarização e variância.

\begin{tabular}{|c|c|c|c|}
\hline & Estimador & Média & Variância \\
\hline \multirow{2}{*}{$1^{\circ}$} & $\mathrm{MQ}$ & -0.0229 & 0.0539 \\
\cline { 2 - 4 } & $\mathrm{AG}$ & $1.58 \times 10^{-4}$ & $2.11 \times 10^{-4}$ \\
\hline \multirow{2}{*}{$2^{\circ}$} & $\mathrm{MQ}$ & -0.1173 & 1.0701 \\
\cline { 2 - 4 } & $\mathrm{AG}$ & 0.0203 & 0.0620 \\
\hline
\end{tabular}

cias da planta, uma vez que a estrutura do modelo (3 zeros e 2 pares de pólos complexos conjugados) e do sistema real são incompatíveis. Porém, o estimador genético produziu uma resposta temporal e frequencial bem semelhante ao sistema real, mostrando-se capaz de captar as dinâmicas principais do sistema real, apesar das limitações de ordem inerentes à estrutura do seu modelo (2 pares de pólos complexos conjugados). Além disso, observa-se também que o modelo obtido pelo estimador dos mínimos quadrados é de fase não mínima (ou seja, apresenta zeros instáveis). O estimador genético não apresenta este problema, uma vez que não é possível se obter zeros fora do espaço de busca. Esta foi uma restrição imposta no trabalho, porém a representação proposta permite que se identifiquem sistemas de fase não mínima através do aumento dos limites do espaço de busca para os zeros.

Também no 2 caso, pode-se perceber que o estimador genético produziu uma resposta mais adequada que o método dos mínimos quadrados como pode ser comprovado nas figuras 5 e 6 . Da análise da tabela 4 , nota-se que em ambos os casos o compromisso entre polarização e variância foi otimizado com o estimador genético.

\section{CONCLUSÃO}

Neste trabalho um estimador de pólos e zeros utilizando algoritmos genéticos foi desenvolvido. O modelo produzido por este estimador possui uma estrutura (ordem)

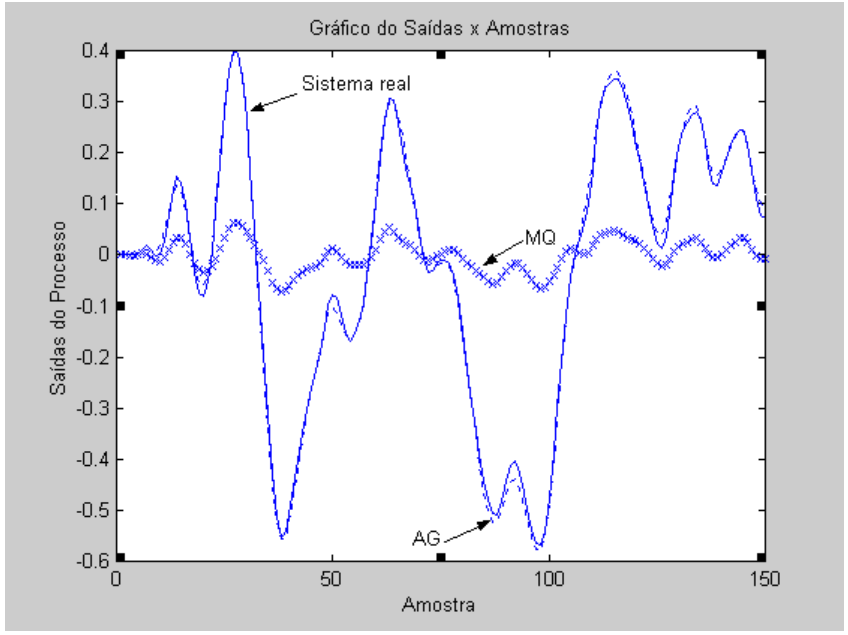

Figura 3: Resposta Temporal

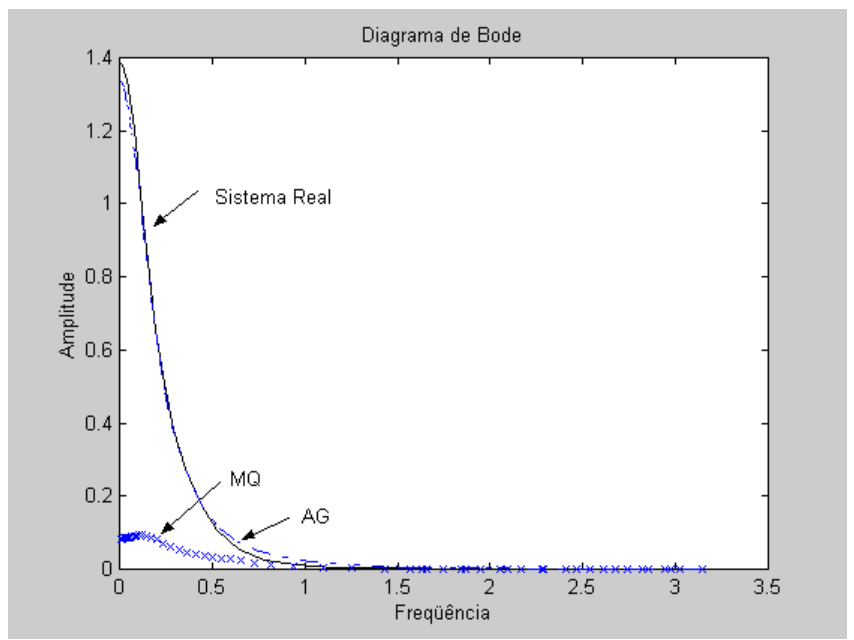

Figura 4: Resposta Frequencial 


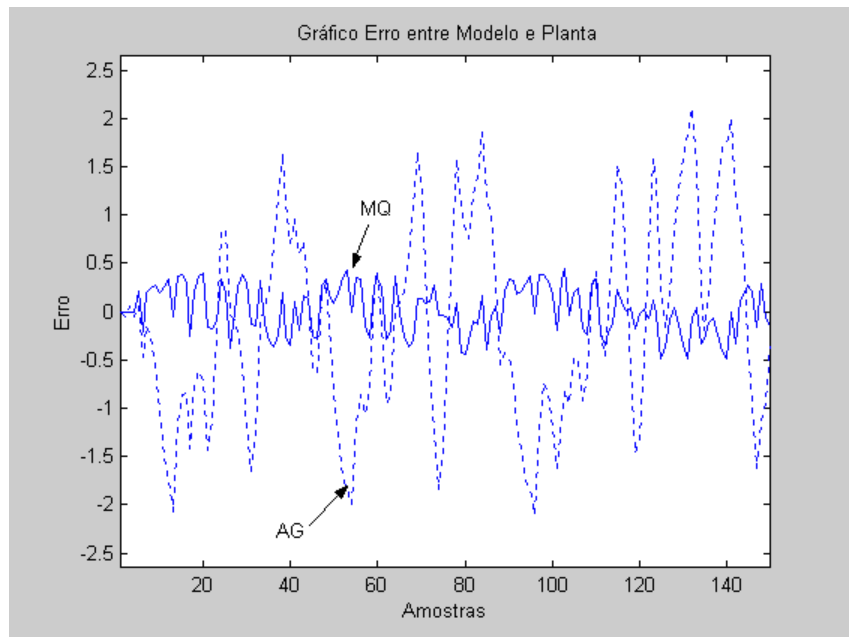

Figura 5: Erro Mismatch

que minimiza o compromisso entre os erros de polarização e variância deste modelo. Isto foi possível graças a escolha da representação dos indivíduos e da função de fitness (ambas inéditas) desenvolvidas para o estimador. As principais vantagens deste estimador são:

- O método é uma alternativa confiável e de fácil utilização aos métodos tradicionais de identificação não-linear de parâmetros que são baseados em busca pelo gradiente e que apresentam problemas de convergência, de mau condicionamento de matrizes hessiana, e de tempo computacional, entre outros.

- Evita-se a aplicação repetida do estimador para diferentes estruturas de modelo a fim de se descobrir a ordem ótima do sistema, isto é, além da estimação de parâmetros, este método fornece diretamente a estrutura do modelo de pólos e zeros a ser utilizado.

Para validação do método, foram realizados estudos de simulação. A partir da análise dos resultados simulados pode-se perceber que o estimador genético produziu resultados satisfatórios quando comparado com o método tradicional dos mínimos quadrados, principalmente na aplicação em sistemas de ordem superior e não lineares.

\section{REFERÊNCIAS}

Arruda, L.V.R.; Fardin, J.F. and Amaral, W.C.(1998). Relevant Identification to Predictive Control Using Genetic Algorithm, Int. Symposium on Engineering of Intelligent Systems, Tenerife, Spain, pp 292-297.

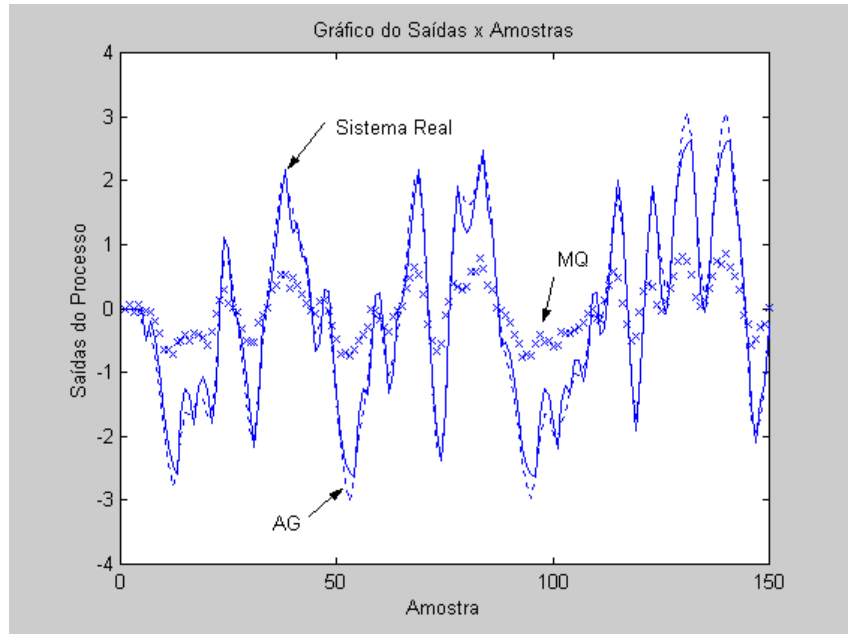

Figura 6: Resposta Temporal

Coelho, L.S. e Coelho, A.A.R. (1999). "Algoritmos evolutivos em identificação e controle de processos: uma visão integrada e perspectivas", $S B A$ Controle e Automação, v.10, n.1, pp.13-30.

Fávaro, S. (1999). Estimação de polos e zeros de modelos usando algoritmos genéticos. Dissertação de Mestrado, CEFET-PR, Curitiba - PR.

Fávaro, S. e Arruda, L.V.R. (2000). Identificação genética de polos e zeros de modelos baseada no compromisso entre os erros de polarização e variância, XIII Congresso Brasileiro de Automática, Florianópolis, SC, pp $98-103$.

Lindskorg, P. (1997). Fuzzy identification from a grey box modeling point of view. In $\mathrm{H}$. Hellendoorn and D. Driankov, editors, Fuzzy Model Identification: Selected Approaches. Springer-Verlag, Heideberg, 1997.

Holland, J.H. (1975). Adaptation in Natural and Artificial Systems. The University of Michigan Press, Ann Arbor, 2nd edition.

Joines, J. A. (1998). Disponível em "www.ie.ncsu. edu / gaot/gatoolbox/gaot".

Kristinsson, K. and Dumont, G.A. (1992): System Identification and control using genetic algorithm, IEEE Trans. On Sys., Man, and Cybernetics, n.22, pp. 1033-1046.

Ljung, L. (1999). System Identification: Theory for the User, 2nd Edition, Prentice - Hall, Englewood Cliffs, NJ. 
Ljung, L. (1985). Asymptotic Variance Expression for Identified Black-Box Transfer Function Models, IEEE Trans. Automatic Control, AC-30, pp. 834844.

Michalewicz, Z. (1996). Genetic Algorithms + Data Structures = Evolution Programs, 3th edition, Springer - Verlag, New York.

Ninness, B. and Goodwin G.C. (1995). Estimation of Model Quality, Automatica, 31, pp. 1771-1797.

Tan, K. C., Li, Y., Murray-Smith, D .J. and Sharman, K. C. (1995). Nonlinear Parameter Estimation via the Genetic Algorithm, Proc. IEE/IEEE Int. Conf. On GA in Eng. System: Innovations and Applications, Sheffield, U.K., pp. 164-169. 